\title{
INNOVACIÓN SOCIAL ABIERTA EN EL DISEÑO DE UNA POLÍTICA Y ESTRATEGIA DE FORMALIZACIÓN SOSTENIBLE: UN CASO COLOMBIANO DE GOBIERNO COLABORATIVO*
}

\author{
IVÁN DARÍO HERNÁNDEZ** \& OSCAR SÁNCHEZ*** \\ UNIVERSIDAD NACIONAL DE COLOMBIA
}

Recibido/ Received/ Recebido: 09/10/ 2013 - Aceptado/ Accepted / Aprovado: 29/05/2014

\begin{abstract}
Resumen
Se presenta este caso de estudio sobre el proyecto de investigación-acción que buscó aportar en la construcción de vías para la solución a la problemática de la informalidad empresarial y laboral en Colombia (2011-2013). Este proyecto utilizó como objeto de estudio la observación y reflexión en el propósito de comprender los determinantes para el éxito o fracaso de un esfuerzo colaborativo, cuando se persiguen objetivos sociales retadores (innovaciones sociales) desde un ámbito mixto (gobierno y sector privado). El proyecto fue desarrollado por el Grupo de Investigación en Economía Evolucionista e Institucional de la Universidad Nacional de Colombia, financiado por Colciencias en alianza con la Cámara de Comercio de Bucaramanga. Se encontró que la capacidad de un equipo interinstitucional para producir resultados que tengan un impacto importante en la sociedad (lograr innovaciones sociales) se construye a partir de la fortaleza de redes interinstitucionales que permiten inferir la emergencia de una nueva metainstitución con la legitimidad, recursos, conocimiento y alineamiento estratégico suficiente para una apropiada armonización de esfuerzos dentro de cada institución.
\end{abstract}

Palabras clave: Innovación social, Innovación abierta, Formalización, Empleo, Gobierno colaborativo.

Artículo de investigación derivado del proyecto "Estrategia de apoyo a la formalización empresarial y laboral a partir de actividades de investigación, innovación y desarrollo tecnológico", el cual fue financiado por Colciencias.

Agradecemos a Colciencias por el apoyo y financiación de la investigación, en particular al exdirector Francisco Miranda, al Subdirector Jorge Cano, Galo Tovar, Andrés Echeverry, Mary Luz Escobar y Adriana Palacios. Agradecemos mucho a la Cámara de Comercio de Bucaramanga por toda su expedita gestión en particular a su Vicepresidente, Iván Ardila, a Carolina Torres, Ana María Plazas y Ricardo Arenas. Queremos agradecer también, muy especialmente, a todas las entidades participantes en el proceso de construcción colectiva relacionada en el anexo correspondiente y, sobre todo, a los empresarios con iniciativas informales que fueron encuestados. Este documento hará parte de un libro que se publicará en el futuro mediato con los resultados completos de la investigación con la participación de todos los investigadores participantes, incluidos también las lecciones aprendidas de la prueba piloto.

** Profesor Asociado, Facultad de Ciencias Económicas, Universidad Nacional de Colombia. Economista - Universidad Nacional de Colombia, MSc Economía - Universidad Nacional de Colombia, MSc Economía - Universidad de Warwick, Ph D. Economía - Universidad de Manchester. Director del Grupo de Economía Evolucionista e Institucional, Universidad Nacional de Colombia. Correo electrónico: idhernandezu@unal.edu.co. Dirección postal: Carrera 64 \# 23a-49, Bogotá - Colombia.

**** Investigador del Grupo de Economía Evolucionista e Institucional, Universidad Nacional de Colombia. Ingeniero Industrial - Universidad del Valle, Especialista en Sistemas de Información en la Organización - Universidad de los Andes, M Sc Administración - Universidad Nacional de Colombia. Correo electrónico: osdsanchezgo@unal.edu.co. Dirección postal: Cra 57 No 11A - 50; Apto 501A, Cali - Colombia. 


\title{
OPEN SOCIAL INNOVATION IN POLICY AND STRATEGY DESIGN FOR SUSTAINABLE FORMALIZATION: COLOMBIAN CASE OF COLLABORATIVE GOVERNMENT
}

\begin{abstract}
This paper shows the results of a case study based on a research project - action to contribute in the construction of pathways to solve business and labor informality in Colombia (2011 - 2013). This study was based on observation and reflection process in order to understand success or failure determinants of a collaborative effort when challenging social objectives (social innovation) from a mixed environment (government and private sector). The project was developed by Research Group on Evolution and Institutional Economics of the Universidad Nacional de Colombia, funded by Colciencias in alliance with the Cámara de Comercio de Bucaramanga. The author found that the capacity of an inter- institutional team to produce results with significant impact on society (achieving social innovations) is built on the strengthen of inter-institutional networks allowing the emergence of a new mega-institution with legitimacy, resources, knowledge and strategic alignment enough to harmonize efforts within each institution.
\end{abstract}

Keywords: Social innovation, Open innovation, Formalization, Employment, Collaborative government.

\section{INOVAÇÃO SOCIAL ABERTA NO DESENHO DE UMA POLÍTICA E DE UMA ESTRATÉGIA DE FORMALIZAÇÃO SUSTENTÁVEL: UM CASO COLOMBIANO DE GOVERNO COLABORATIVO}

\begin{abstract}
Resumo
Apresentam-se os resultados de um estudo de caso originado do desenvolvimento de um projeto de pesquisa-ação que procurou contribuir na construção de vias para a solução à problemática da informalidade empresarial e laboral na Colômbia (2011-2013). O objeto de estudo foi realizar processos de observação e reflexão com o propósito de compreender os determinantes para o sucesso ou fracasso de um esforço colaborativo quando se perseguem objetivos sociais desafiadores (inovações sociais) desde um âmbito misto (governo e setor privado). O projeto foi desenvolvido pelo grupo de pesquisa em Economia Evolucionista e Institucional da Universidade Nacional da Colômbia, financiado por Colciencias em aliança com a Câmara de Comércio de Bucaramanga. Encontrou-se que a capacidade de uma equipe interinstitucional para produzir resultados que tenham um impacto importante na sociedade (conseguir inovações sociais) constrói-se a partir da fortaleza de redes interinstitucionais que permitem inferir a emergência de uma nova meta-instituição com a legitimidade, recursos, conhecimento e alinhamento estratégico suficiente para uma apropriada harmonização de esforços dentro de cada instituição.
\end{abstract}

Palavras chave: Inovação social, Inovação aberta, Formalização, Emprego, Governo colaborativo.

Hernández, I. \& Sánchez, O. Innovación social abierta en el diseño de una política y estrategia de formalización sostenible: un caso colombiano de gobierno colaborativo. En: Revista de la Facultad de Ciencias Económicas de la Universidad Militar Nueva Granada. rev.fac.cienc.econ, XXII (2).

JEL: O31, O32, O39. 


\section{Introducción}

Dado que Colombia se encuentra en un proceso de desarrollo económico con ambiciosos objetivos gubernamentales en términos del mejoramiento de la calidad de vida de los ciudadanos y la promesa de ampliación de cobertura y mejoramiento de calidad de los servicios públicos más esenciales, se presentan los resultados de este estudio que indagó sobre la naturaleza de las innovaciones sociales y su relación con el concepto de gobierno colaborativo, teniendo de fondo como supuesto la idea que se requieren transformaciones importantes en las estructuras actuales para lograr alcanzar dichas metas, las cuales no se podrán realizar mediante simples mejoras de los modelos existentes.

La investigación se ha centrado en la problemática del empleo informal en Colombia y para ello se ha desarrollado un caso de estudio que analiza una sucesión de procesos colaborativos encaminados a convertir la reducción del empleo informal a través de la formalización sostenible en una innovación social.

\subsection{La informalidad laboral y empresarial; un tema importante para Colombia}

Este año se cumplen 30 años de instaurada la Misión de Empleo en Colombia dirigida por el destacado académico Hollis Chenery, asesorado por un importante equipo de colaboradores (Chenery, Ocampo \& Ramírez 1986). El economista José Antonio Ocampo, en ese entonces, afirmó que era la primera vez que se contaba con "una visión global del problema laboral en Colombia dentro del contexto macroeconómico" (Revista Semana 1986). Como integrante de dicha Misión, el especialista en mercado laboral, Hugo López diagnosticó el problema de informalidad como uno asociado con los bajos ingresos, marginalidad jurídica, social y, en especial, de las normas de seguridad social (López, 1986). Claro está que el problema no se circunscribe solamente a la realidad colombiana actual sino que es un problema que se reconoció en la última reunión del G20 como uno que afecta las economías a nivel mundial (Simsek, 2014). De tal manera que si no se aplican correcciones pertinentes, es de esperarse que la siguiente crisis profundice el problema ${ }^{1}$.

En efecto, la situación es susceptible de empeorar dado que algunas investigaciones han demostrado que el fenómeno de informalidad es reincidente una vez se termina la etapa de auge, etapa en la que se encuentran actualmente Latinoamérica y el Caribe (Perry et. al, 2007; Maloney, 2003). La Misión de Chenery tuvo una gran influencia sobre las reformas y leyes subsecuentes, no sólo a partir del 2010 sino también en las dos décadas anteriores ${ }^{2}$. En dicha tradición subyace latente una disyuntiva: la elección entre políticas macroeconómicas globales o, de otro lado, políticas específicas sectoriales (como las reformas a la legislación laboral de 2010 y 2012 mencionadas anteriormente). Desde el punto de vista teórico, las políticas macroeconómicas del lado de la demanda o de corte keynesiano han sido concebidas como separadas de las políticas del lado de la oferta de instrumentos de regulación del mercado laboral (González, 2003). El caso estudiado es un proyecto de investigación-acción que tiene como objeto una posible resolución sostenible al problema de la informalidad y su respectiva reincidencia.

\section{3 ¿Por qué la acción colectiva para la resolución al problema de informalidad?}

Desde los trabajos seminales de Hart sobre informalidad hace más de 40 años (Hart, 1973), se han desarrollado diversas corrientes de pensamiento frente a la informalidad (Gerxhani, 2004). Ciertas corrientes entienden la informalidad como una que hay que eliminar o regular, mientras que otra corriente entiende la informalidad como un grupo vulnerable

1 Comparada con otras regiones de ingresos medios, América Latina y el Caribe está casi veinte puntos por encima de la tasa promedio de $37 \%$. Esto la hace la región con las tasas más altas del mundo: 56 \% de trabajadores informales según cifras del Banco Interamericano de Desarrollo durante su asamblea en 2013 (BID).

2 El desaparecido e influyente economista Juan Luis Londoño, secretario técnico de la Misión Chenery, lideró reformas de seguridad social y de flexibilización laboral en los 90's y 2000's. 
y que debe ser asistido a través de políticas sociales. Otra la entiende como empresarios que deben ser liberados de la regulación del gobierno, mientras que otra perspectiva entiende a la fuerza informal como productores y trabajadores desprotegidos que necesitan ser cubiertos por la legislación laboral (Chen, 2007).

Debido a la existencia de toda esta gran cantidad de puntos de vista es recomendable definir el problema de la informalidad dentro de un contexto social y cultural. Sin embargo, el debate se ha desarrollado sin atender esta variedad. Ha existido una visión dominante y excluyente debido a la fuerte influencia que ejerció la Misión Chenery en Colombia y el programa Doing Business del Banco Mundial, entre otras tradiciones similares (Maloney, 2004). Dicha tradición se concentra en bajar los costos de formalizar empresas y trabajadores y se encuentra en el espíritu de las reformas de regulación tributaria y laboral recientes. A partir del 2010, esfuerzos como la Ley de Formalización y Generación de Empleo de 2010 y la Reforma Tributaria del 2012 en Colombia, pretenden contribuir a la disminución de la informalidad en el país bajando los costos laborales de contratación por medio de la reducción de la carga parafiscal. La buena intención, en principio, es reducir la desigualdad, como subraya el actual Ministro de Hacienda Mauricio Cárdenas, permitir la formalización de un millón de empleos y reducir la informalidad. El Ministro, en su previa calidad de investigador junto con otros investigadores de la Universidad de Los Andes y Fedesarrollo, han difundido consecuentemente los fundamentos teóricos y sustento empírico que conllevaron a dicha reforma (Cárdenas \& Rozo, 2009; Cárdenas \& Mejía, 2007; Santamaría \& Rozo, 2009).

Además de estos estudios y su respectiva evidencia, se presentó un debate previo que presentó insuficientes puntos de vista y cuestionamientos de corte académico a la visión que inspira las reformas (CEDE, 2011). Bajo esta perspectiva, parece un debate agotado, con pruebas contundentes y que pareciera que no requiriera más debate. Nada más lejos de la realidad. En una investigación sobre las instituciones comparadas en Latinoamérica (Hernández, 2012a), se enfatizó que el problema no está en que la élite de las últimas décadas en la administración de política económica en Colombia se haya autolegitimado como inmutable a intereses creados por la politización. Por lo contrario, nada tiene pretender distanciarse de la clase política si la convicción es que la toma de decisiones es de corte puramente técnico. Sin embargo, se presentan ante la opinión pública con políticas únicas, y señalan cualquier cuestionamiento como posiblemente contaminado por dichos intereses políticos. De allí que la política económica en Colombia sea objeto de poco debate, pues entraría en contradicción con los profundos valores arraigados de neutralidad a la política. Bajo esta concepción, la política económica es una sola, inmune y despolitizada.

El debate sobre informalidad y su reincidencia no sólo no está agotado sino que es inadecuado apostarle solamente a una visión, supuestamente inmune y despolitizada, que ignore los diferentes significados e implicaciones que puedan tener para los distintos sectores socioculturales. Por supuesto, no se excluyen los sectores que se identifican con la visión dominante, pero entonces no serán los únicos invitados. Es por esto que en el caso estudiado se observó la influencia de la literatura de desarrollo deliberativo aplicando las metodologías de la investigación-acción (Fals-Borda, 1998), las cuales recurren a la estructura social para la definición del problema de desarrollo respectivo (Portes, 2009, 2007; Morrison \& Singer, 2007). En pocas palabras, este cuerpo teórico, inspirado en el institucionalismo (Guha-Khasnobis, Kanbur \& Ostrom, 2007) recomienda que se convoque a los dolientes relacionados con el problema (incluidos desde los beneficiarios/usuarios hasta la tecnocracia que implementará las recomendaciones resultantes) para que, por medio de metodologías de construcción social (i.e. gestión del conocimiento), sean las propias instituciones y organizaciones quienes construyan y definan los conceptos y su relación, para el caso, con la informalidad.

\subsection{La innovación social}

En los países desarrollados (Goldenberg, Kamoji, Orton, \& Williamson, 2009), así como en los países en desarrollo (Rodríguez \& Alvarado, 2008) se está avanzando en la comprensión del concepto de la innovación social. El término innovación social 
se ha ligado a la solución de los problemas sociales más complejos (Moore \& Westley, 2011). Tiene que ver con provocar cambios profundos en estructuras sociales complejas, logrando que de dichos cambios emerja una mejor situación en algún aspecto importante para la vida de grupos poblacionales que normalmente se encuentran en una posición adversa en el goce efectivo de sus derechos.

En Iberoamérica, los gobiernos y organismos multilaterales han identificado la innovación social como una vía alternativa de desarrollo más allá de la implementación de políticas sociales por los gobiernos (Rodríguez \& Alvarado, 2008) y los académicos están documentando experiencias exitosas para aprender y encontrar los patrones que permitan su implementación efectiva; por ejemplo los estudios de Novy \& Leubolt (2005) en Brazil y Zurbano (2008) en Euskadi.

Un buen número de investigadores se han interesado por clarificar el concepto. Por ejemplo la propuesta de Mulgan $(2006,146)$ :

\section{“(...) La innovación social se refiere a ac- tividades y servicios innovadores que son motivados por el objetivo de satisfacer una necesidad social y que son predominante- mente difundidas por organizaciones cuyo propósito principal es también social".}

Otros estudiosos han aportado sus propuestas para la definición de innovación social, éstas fueron citadas en Cahill (2010):

- The Young Foundation:

"Nuevas y mejores formas de satisfacer apremiantes necesidades insatisfechas".

- Centro para la Innovación Social (Toronto): "Se refiere a nuevas ideas que resuelven retos existentes en lo social, cultural, económico y ambiental para el beneficio de la gente y el planeta. Una verdadera innovación social modifica sistemas, altera permanentemente las percepciones, comportamientos y estructuras que previamente dieron lugar a esos retos".
- Centro para la Innovación Social (Nueva Zelanda): "El diseño e implementación de nuevas formas de satisfacer necesidades sociales".

El estudio de Pol \& Ville (2009) destaca como "muchos investigadores contemporaneos declaran que para entender las fuentes de la innovación se debe entender el milieu en el cual la creatividad tiene lugar. Debe haber un ambiente que conduzca a la creación de nuevas ideas y un contexto en el cual una nueva idea es socialmente innovadora". La tarea de los innovadores sociales es comprender y modificar los sistemas sociales que crean y sustentan los problemas o retos sociales a resolver (Bloom \& Dees, 2008).

Pol \& Ville (2009) utilizan también la analogía del ecosistema y cómo el innovador social debe "cultivar el ecosistema". Esto es: identificar actores claves del sistema social y moldear las relaciones y estructuras para el propósito que se persigue.

La innovación social requiere incrementalmente el uso de redes diversas (Nambisan, 2009) que proveen mejores soluciones de lo que son capaces las entidades independientes. Para que emerja la colaboración se requieren conjuntos de prácticas y sistemas que el autor llama "Plataformas" y de las cuales podría haber de: exploración, experimentación y ejecución.

El estudio de Moore \& Westley (2011) establece una conexión entre las redes de personas y la innovación social; según sus conclusiones, los problemas complejos en la sociedad se resuelven mediante soluciones complejas que implican la reestructuración de las redes existentes a través de mecanismos de intercambio de conocimiento y recursos, construcción $\mathrm{y}$ fortalecimiento de relaciones $\mathrm{y}$ reconocimiento de patrones que mantienen la rigidez institucional.

Los arreglos institucionales (normativos) refuerzan o restringen la generación de redes sociales productoras de capital social para sus integrantes, este capital social es catalizador de otros tipos de capital que conducen al progreso de las regiones (Méndez, Michelini \& Romeiro, 2006) como un ejemplo de innovación social. 
El cambio social a gran escala requiere de una extensiva coordinación intersectorial (Kania \& Kramer, 2011). Este tipo de cambio de gran impacto en la sociedad que resuelve problemas complejos no se puede lograr gracias a esfuerzos heróricos de individuos, ni siquiera gracias a esfuerzos aislados de instituciones privadas o públicas; requiere una agenda común para todos los participantes (alineamiento estratégico en un nivel superior al organizacional), sistemas de medición compartidos, división del trabajo sinérgica, comunicación continua y una organización de soporte independiente a las organizaciones participantes inclusive con su propio talento humano (Kania \& Kramer, 2011).

Según el estudio de Sánchez (2013), los procesos de colaboración en el sector público responden a diferentes tipos de objetivos, algunos son objetivos menores (cuando se mejora alguna capacidad organizacional), otros a objetivos superiores (cuando se crea un nuevo servicio público o se mejora alguno existente o a objetivos muy superiores (cuando se articulan redes de servicios para resolver problemáticas muy complejas de la sociedad); esto es, alcanzar innovaciones sociales. La capacidad de alcanzar unos u otros objetivos está en relación directa con la fortaleza de la red interinstitucional que soporte el proceso colaborativo y a su vez los repetidos procesos colaborativos construyen dicha fortaleza mediante la generación progresiva de confianza entre los actores, conocimiento compartido, normas y sistemas de información y comunicación compartidos (Sánchez, 2013).

También Swyngedouw (2005) identificó la emergencia de arreglos interinstitucionales para la definición de política pública como elementos que compiten $e$ incluso pueden rivalizar con las formas de gobierno típicamente establecidas centralizadamente desde las instituciones públicas para crear nuevas formas de gobierno y nuevos actores promoviendo la innovación en el gobierno.

Aunque no hay un consenso sobre la definición de innovación social (Pol \& Ville, 2009), este estudio se centra en las transformaciones de sistemas sociales que producen reacomodos en las relaciones de los actores generando bienestar en uno o varios grupos poblacionales. Así pues la innovación social consiste en lograr la comprensión colectiva de patrones en los sistemas sociales que generan retos a resolver $y$ diseñar e implementar soluciones que modifican dichos sistemas sociales logrando superar los retos identificados; esas soluciones son complejas y por tanto escasas.

La comprensión de los patrones sociales, el diseño de las soluciones y la implementación de dichas soluciones son procesos sociales colectivos que requieren acción colectiva como ocurre en los procesos de gobierno colaborativo (Sánchez, 2013).

\subsection{El gobierno colaborativo}

Los procesos de construcción colaborativa en el sector público han recibido atención incremental de los investigadores en el campo de la administración pública en la últimas dos décadas; algunos ejemplos recientes: Cooper, Bryer,\& Meek (2006); Dagnino (2004); Emerson, Nabatchi \& Balogh (2012); Johnston, Hicks, Nan \& Auer (2011); McGuire (2006); Sirianni (2008); Zhang \& Cao (2010); sin embargo hay evidencia que demuestra que la práctica de la gestión pública colaborativa no es algo nuevo (McGuire, 2006) y puede ser trazada siglos atrás.

Mediante estos procesos, las entidades del estado pretenden vincular dentro de sus procesos misionales a diferentes actores de la sociedad que por diversas razones pueden llegar a hacer aportes de valor para la obtención de resultados mucho más efectivos que si fueran desarrollados únicamente por la entidad competente; entre otros, los actores pueden ser: grupos o individuos ciudadanos, otras entidades públicas, universidades $u$ otras entidades; en resumen, diversos involucrados que puedan aportar en la construcción, debido a que poseen conocimiento especializado (Hawamdeh, 2007), conocen la perspectiva histórica del asunto en cuestión, cuentan con recursos valiosos, representan puntos de vista interesantes, $\mathrm{u}$ otras diversas razones que este estudio pretende identificar (Johnston et al., 2011). A pesar de lo anterior, se ha reportado que aún el grueso del trabajo de los administradores públicos sigue realizándose mediante sus jerarquías individuales (Agranoff, 2006), lo cual plantea un potencial importante para el desarrollo de éste tipo de gestión pública. 
Gobierno interactivo es el término utilizado por Edelenbos (2005) para ilustrar los procesos de participación ciudadana en el gobierno. En su estudio sobre el caso holandés, Edelenbos establece una tensión entre los organismos oficiales y lo que él denomina proto-instituciones que surgen de los procesos interactivos de gobierno. A partir de sus observaciones de estas instituciones emergentes, determina que a menudo tienen "métodos, etapas, reglas y roles para los actores" generando una estructura emergente.

Como conclusión del caso holandés, hay un "eslabón perdido institucional" en el que se desconecta el proceso de gobierno interactivo del proceso formal de toma de decisiones en la entidad pública. Cuando el proceso de gobierno interactivo termina, las entidades formales recuperan el papel protagónico dejando a las instituciones emergentes -según el discurso de Edelenbos (2005)- detrás de escena.

La gestión pública colaborativa es descrita por O'Leary, Gerard \& Bingham (2006) de la siguiente manera:

\section{“(...) es un concepto que describe el proce- so de facilitar y operar arreglos multiorgani- zacionales para resolver problemas que no pueden ser resueltos fácilmente por organi- zaciones individuales".}

Si bien en su artículo, estos autores no definen un modelo, su definición de gobierno colaborativo permite deducirlo; para ellos la gestión pública colaborativa se trata fundamentalmente de facilitar y operar arreglos multi-organizacionales que son capaces de resolver problemas que las organizaciones individuales por su cuenta no serían capaces de resolver.

De esta definición es destacable que hay un privilegio por el arreglo "multi-organizacional" que podría ser equivalente a "inter-institucional" sobre el proceso mismo de resolución colaborativa de problemáticas. Queda cierta inquietud sobre el verbo "facilitar" que implica la disposición de ciertas condiciones para que el arreglo multi-organizacional emerja versus la posibilidad de haber utilizado el verbo "crear" que implica el involucramiento directo y el compromiso con su emergencia.
De cualquier forma, esta definición permite proponer claramente dos fases en el marco del gobierno colaborativo; una fase de crecimiento del arreglo de entidades en la que el objetivo es facilitar las condiciones para el desarrollo de éste, y una segunda fase en la que el arreglo multi-organizacional debe ser operado, de tal forma que puedan ser resueltos colaborativamente las mencionadas situaciones problemáticas. Nada nos dice este modelo sobre si el arreglo multi-organizacional puede ser operado múltiples veces para la solución de múltiples problemas o por el contrario, cada problema tiene su propio y único arreglo multi-organizacional que debe ser creado.

Para Ansell \& Gash (2007), el gobierno colaborativo se define así:

"Un arreglo gubernamental donde una o más entidades públicas se involucran directamente con interesados no estatales en un proceso colectivo de toma de decisiones que es formal, orientado al consenso, $y$ deliberativo y que busca elaborar o implementar políticas públicas o dirigir programas o bienes públicos”.

Según esta definición, el gobierno colaborativo se trata de un arreglo de entidades públicas que se involucran en un único proceso de toma de decisiones de manera colectiva. Para estos autores, otros esfuerzos que no sean orientados a la toma de decisiones no hacen parte del concepto de gobierno colaborativo. Sin embargo, el modelo que proponen Ansell \& Gash $(2007,550)$, se centra en el proceso de colaboración para la toma de decisiones como elemento principal, entendiendo que el logro de sus objetivos es facilitado por: unas condiciones iniciales en términos de las relaciones de poder de los participantes (Agranoff, 2006) y la historia previa de cooperación o conflicto entre ellos, unas reglas de juego (diseño institucional) de la colaboración y un estilo de liderazgo que está en capacidad de facilitar que la colaboración emerja. Desde esta perspectiva, es posible comprender que para Ansell \& Gash $(2007,550)$ el arreglo gubernamental está embebido en cada proceso colaborativo y de ésta manera es único y no reutilizable. 
Así, para ellos gobierno colaborativo resulta ser un proceso colaborativo de toma de decisiones que cuenta con un arreglo gubernamental de entidades públicas para la solución de un problema particular. Otros modelos parecen ser más acertados para explicar el fenómeno. La siguiente es la definición de Emerson et al. $(2012,2)$ para el gobierno colaborativo:

"Son procesos y estructuras de toma de decisiones de política pública y gestión que involucran personas constructivamente a través de los límites de agencias públicas, niveles de gobierno y/o las esferas pública, privada y cívica para llevar a cabo un propósito público que de otra forma no podría realizarse".

Es notable la diferencia entre esta y la propuesta de Ansell \& Gash (2007); mientras los segundos se concentran en un proceso de colaboración para la toma de decisiones; Emerson et al. (2012) se concentran no en el proceso sino en el arreglo de entidades involucradas en el proceso. Este modelo muestra una perspectiva más amplia sobre el gobierno colaborativo donde los "procesos" existen y se denominan "acciones", solo que son parte de una construcción más compleja que incluye: un régimen de gobierno colaborativo, la dinámica de colaboración, un circuito de retroalimentación, las acciones y los resultados; todo en el marco de su entorno de referencia. A su vez al llamar este entorno como "el entorno del sistema" los autores declaran que entienden el gobierno colaborativo como un sistema, lo cual es una característica nada despreciable para entender su visión del gobierno colaborativo y por tanto este modelo se considera en el marco de esta investigación como el más apropiado para la comprensión del fenómeno.

El modelo evolutivo de gobierno colaborativo (Sánchez, 2013) relaciona directamente en un plano, el grado de complejidad de los objetivos que un esfuerzo colaborativo pretende alcanzar con la fortaleza de la red interinstitucional requerida para el logro de dichos objetivos; lo componen:

- Los ejes del plano que corresponden a: tipo de objetivos (menor, superior, muy superior) y la fortaleza de la red organizacional interinstitucio- nal que se mide cualitativamente como una variable compuesta por elementos como el número de contactos previos, la propensión a compartir conocimiento, la existencia de sistemas de información compartidos y el surgimiento de normas de comportamiento aceptadas colectivamente (Sánchez, 2013).

- Las áreas: área de los objetivos menores (se pretende habilitar una capacidad al interior de una organización para la prestación de un servicio público), área de los objetivos superiores (se busca habilitar un nuevo servicio público o mejorar sustancialmente su calidad, cobertura $\mathrm{u}$ oportunidad) y área de los objetivos muy superiores (se espera habilitar una cadena de servicios públicos prestados conjuntamente entre varias instituciones para la satisfacción de una necesidad).

- Las zonas: la zona de inviabilidad (aquella zona del plano en la que la fortaleza de la red interinstitucional no es suficiente para el logro de los objetivos), la zona de los esfuerzos innecesarios (donde la fortaleza de la red es superior a lo mínimo requerido para alcanzar los objetivos trazados) y la zona de la razonabilidad (la región del plano en la que es factible que se alcancen los objetivos dado un nivel de fortaleza de la red interinstitucional requerida).

- Conglomerados: en el estudio de Sánchez (2013) se identificaron 5 conglomerados o grupos de datos que convergieron según su caracterización en variables de interés para el estudio de la emergencia de la colaboración en este tipo de procesos; además el modelo se completa con un sexto conglomerado teórico. Los conglomerados definen la zona de razonabilidad.

\section{La definición del problema y su método}

La pregunta de investigación interrogó sobre la naturaleza de las innovaciones sociales abiertas, intentando dilucidar a partir de un caso real, las complejidades de éste fenómeno y su relación con el constructo del gobierno colaborativo. Esta es: ¿Cómo se 
pueden lograr innovaciones sociales desde un ámbito público-privado?

Se escogió el método de estudio de caso como estrategia de investigación dado que esta estrategia permite obtener conclusiones sobre fenómenos complejos haciendo generalizaciones conceptuales, facilita dar respuesta a preguntas de investigación del estilo ¿Cómo? o ¿Por qué?, para la comprensión de un fenómeno contemporáneo para el cual no es posible controlar sus condiciones de comportamiento o su entorno (Yin, 2003).

Se siguió un proceso de investigación-acción en el que uno de los investigadores principales dirigió el proyecto que se utilizó como caso de estudio y el segundo investigador participó de las sesiones de trabajo como observador.

Se formularon dos marcos conceptuales: en innovación social y en gobierno colaborativo. Los procesos colaborativos que se siguieron en el marco del proyecto se analizaron a la luz de la teoría para mejorar sus probabilidades de éxito y a su vez se verificaron hipótesis teóricas en la práctica. En relación con la comprensión del proceso colectivo interinstitucional, se acudió a la observación de las dinámicas del proyecto, la revisión documental y la contrastación de un modelo teórico previamente establecido en Sánchez (2013).

Las observaciones se documentaron en unas notas de campo que luego fueron articuladas mediante una síntesis integral con todos los documentos producidos durante el proyecto y con base en las reflexiones de los investigadores se generaron unas conclusiones.

\subsection{Observaciones}

\subsubsection{El caso de estudio}

Se ha realizado un proceso de investigación-acción en el marco del proyecto financiado por Colciencias y en alianza con la Cámara de Comercio de Bucaramanga para la formalización laboral y empresarial a partir de actividades de investigación, innovación y desarrollo tecnológico entre 2011 y 2013 en el departamento de Santander, Colombia ${ }^{3}$. Su objetivo fue: aunar esfuerzos para estructurar y pilotar una estrategia de apoyo a la formalización empresarial y laboral que genere condiciones para la innovación y el desarrollo productivo del país.

\subsubsection{Descripción de los tres esfuerzos de colaboración}

Este proyecto se adelantó en tres fases (Gómez, 2013). La primera fase del proceso tuvo dos frentes de trabajo, el primero se centró en establecer el Comité Asesor Regional (CAR) conformado por representantes de entidades relevantes del entorno institucional santandereano. La labor del CAR consistió en dar patrocinio al proceso al interior de las entidades así como servir de órgano rector del esfuerzo colaborativo. Además se conformaron cuatro subcomités para trabajar en la construcción colectiva del concepto de informalidad a través de la definición de las dimensiones del constructo multidimensional, atendiendo a las estructuras sociales existentes (Sánchez, 2013):

- Subcomité Doing Business: Integrado por entidades con visión técnica y que históricamente han tenido metas correlacionadas negativamente con las iniciativas informales y su perspectiva histórica de la solución está relacionada con el registro mercantil de las iniciativas, el pago de impuestos y el respeto por la propiedad intelectual entre otros.

- Subcomité Institucional y de Desarrollo Productivo: Integrado por entidades con visión técnica pero que su naturaleza les impone metas relacionadas positivamente con el desarrollo económico formal e informal.

3 El proyecto arrojó como resultado 23 documentos escritos elaborados entre diez investigadores en más de 1.200 páginas bajo la dirección del primer autor del presente artículo; se convocaron 25 entidades en más de 20 reuniones; y se entrevistaron más de 200 iniciativas informales recabadas de una base de datos con más 900 entradas. En el blog www.labs-ci.com/wikiccb y el sitio web www. soyformal.com se puede obtener más información sobre el proceso y productos de investigación. 
- Subcomité de Competitividad Empresarial: Integrado por entidades que tienden a concentrarse más en conseguir los beneficios de la formalización que en los métodos empleados para lograrla.

- Subcomité de Prospectiva en Formalización e Innovación: Integrado por entidades que tienen una visión macroscópica del fenómeno de la informalidad a través de la reflexión y discusión de estudios y políticas.

El segundo frente de trabajo de la primera fase consistió en la elaboración de varios documentos que aportaron al fortalecimiento conceptual del proyecto y a la determinación de las posibilidades normativas y políticas disponibles en Colombia para la formalización empresarial y laboral vía innovación. Estos documentos fueron desarrollados por investigadores y consultores expertos: Cabal (2011); Castro (2012a, 2012b, 2012c, 2012d, 2012e, 2012f); Hernández (2011, 2012b, 2013); Taborda (2011a, 2011b, 2011c, 2011d, 2011e).

La fase 2 consistió en la definición de la estrategia propiamente dicha (Gómez, 2013); ésta estrategia consistió en establecer para el caso del departamento de Santander un pareo entre los instrumentos de apoyo a la formalización mediante actividades de innovación y las expectativas y necesidades de los empresarios informales (Cabal, 2011; Hernández, 2012b, 2013).

Gráfica 1. Estrategia de pareo entre oferta y demanda para la formalización sostenible mediante la innovación ${ }^{4}$

\section{Oferta}

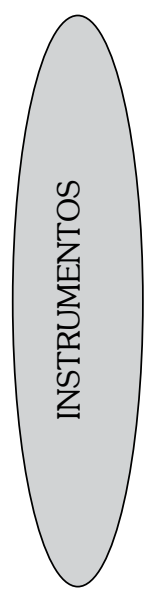

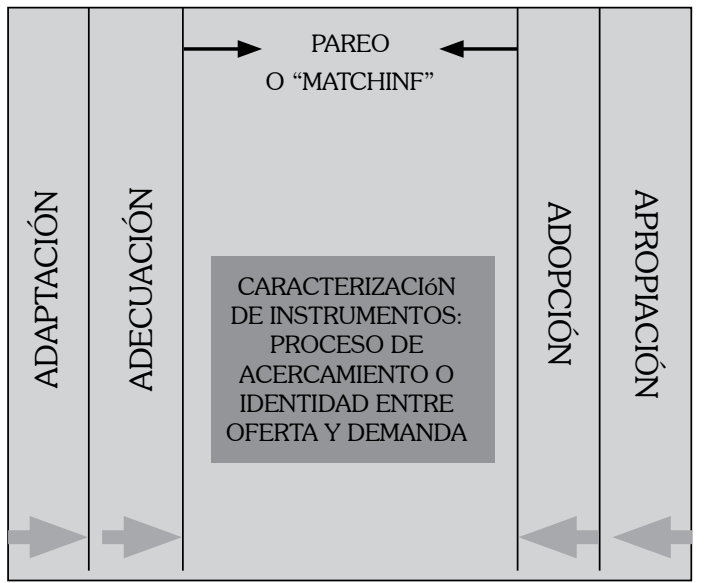

\section{Demanda}

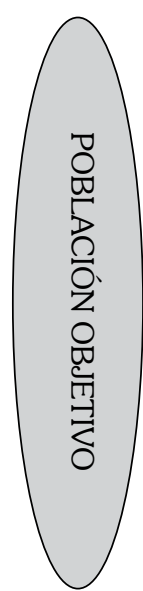

Esta estrategia consistía en eliminar el patrón identificado de baja adopción de los instrumentos de política pública por parte de los empresarios (población objetivo), para esto se diseñó un proceso de ambos lados que consistió en la adaptación de los instrumentos y su adecuación para propiciar la apropiación y final adopción de éstos de parte de los empresarios. El foco fue en instrumentos que redujeran la probabilidad de reincidencia de los empresarios en la informalidad por lo que se le llamó a esta estrategia: Formalización Sostenible Mediante Innovación - FSMI.

La tercera fase consistió en la implementación de un piloto para la implementación de la estrategia de pareo en un caso particular, de tal manera que se

4 Fuente: Hernández (2012b, 2013). 
pudieran obtener aprendizajes tendientes a la propuesta final de políticas, estrategias e instrumentos de apoyo a la formalización empresarial y laboral a partir de actividades de investigación, innovación y desarrollo tecnológico. El piloto se adelantó en cuatro fases: Fortalecimiento del Comité Asesor Regional (CAR), Socialización y Contextualizacón de los empresarios, Adaptación y Adecuación de los instrumentos y Capacitación y Asistencia Técnica. Como resultado del piloto se estableció el sitio web www. soyformal.com que cuenta con información relevante sobre la estrategia de formalización, un formulario para que los empresarios soliciten ser contactados por la iniciativa y todos los documentos producidos durante la primera fase del proyecto.

Como resultado del piloto, (Gómez, 2013) estableció que:

- La figura del Comité Asesor Regional (CAR) como organismo patrocinador, empoderado por las instituciones participantes es fundamental para lograr la coordinación interinstitucional.

- El organismo patrocinador y coordinador debe nombrar un Gerente del esfuerzo colaborativo.

- Para los esfuerzos colaborativos es indispensable tener un modelo de sostenibilidad financiera.

- La optimización del tiempo de los empresarios fue un factor clave. Dado que éstos cumplen toda clase de funciones en sus empresas, para ellos el tiempo es un recurso escaso y la disposición de los servicios en horarios que se adapten a sus restricciones es un aspecto clave.

- Resulta valioso establecer mecanismos de coordinación operativa entre las entidades participantes y el esfuerzo colaborativo de cara a la población objetivo.

- Dado que las unidades empresariales normalmente son muy pequeñas, los límites entre la empresa y la familia están difuminados por lo que los instrumentos de intervención, así como los consultores que los aplican deben estar conscientes de esa realidad.

\subsubsection{La estrategia de formalización como punto de desencuentro entre los actores del esfuerzo colaborativo}

Durante la formulación de la estrategia fueron evidentes los disensos y discusiones sobre cuál debería ser la naturaleza de esta, cuáles sus focos y sobre todo cuáles los resultados a esperar a partir de su implementación. Estas discusiones generaron tensión e hicieron más lento el proceso, pero al estar en la mesa los actores sociales involucrados, estas discusiones tempranas enriquecieron el resultado y aportaron legitimidad a las conclusiones y definiciones de la formulación estratégica.

\section{Resultados}

\subsection{La innovación abierta: los esfuerzos de colaboración en el marco del modelo evolutivo de gobierno colaborativo}

Según el modelo evolutivo del gobierno colaborativo (Sánchez, 2013), en la medida que los objetivos que se plantea un proceso/esfuerzo colaborativo incrementan su complejidad, se requiere mayor fortaleza interinstitucional en red para alcanzarlos. El caso de las innovaciones sociales es un caso extremo de colaboración en el que se alcanzan objetivos del nivel más alto; esto es: transformar un sistema social para lograr un mejor desempeño en alguna variable de interés y en este caso específico, lograr la formalización empresarial sostenible de las microempresas informales en Santander. Esta innovación social se planteó como una sucesión de esfuerzos colaborativos (innovación social abierta) que hasta este punto consistieron en:

- La constitución de la plataforma de coordinación (Comité Asesor Regional) y la definición colectiva de la problemática a abordar (formalización empresarial sostenible mediante la innovación).

- La formulación de la estrategia.

- El piloto para probar y refinar la estrategia.

Otros procesos colaborativos se definirán en el futuro a partir de estos tres primeros para asegurar que continuamente se incrementa la fortaleza inter-insti- 
tucional en red y a su vez es posible aspirar a objetivos más complejos hasta el logro de la innovación social.

Tanto la conformación del CAR y la definición colectiva de la problemática como la formulación de la estrategia de formalización, son procesos colaborativos que se pueden categorizar en el conglomerado 3 (según el modelo evolutivo de gobierno colaborativo), principalmente por la dependencia que presentan de un programa o proyecto central que provee los recursos para su desarrollo y porque se limitan al fortalecimiento institucional sin un impacto real en la prestación de servicios públicos.

Por otro lado, la realización del piloto encaja en el conglomerado 1 dado que es un proceso que depende de un proyecto de orden superior y mejora la prestación de servicios actuales (aunque en modo de piloto). La trayectoria trazada entre los procesos colaborativos 1, 2 y 3 muestra una evolución que permite prever un continuo avance en fortaleza interinstitucional en red y así el potencial de crecimiento para lograr la innovación social.

Gráfica 2. Modelo evolutivo de gobierno colaborativo - El caso de FSMI en Bucaramanga ${ }^{4}$

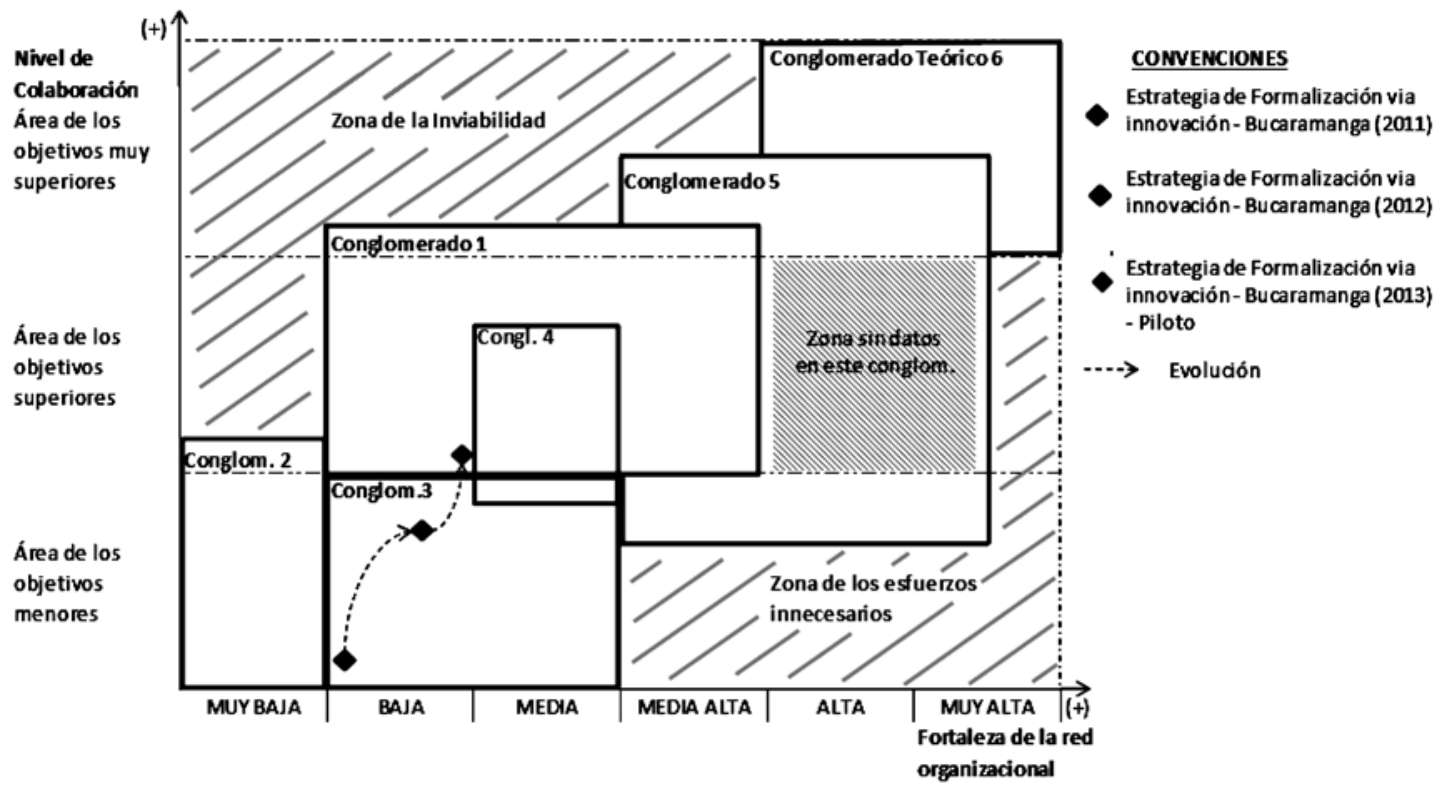

\section{Conclusiones}

Las innovaciones sociales se pueden comprender desde una perspectiva evolutiva y no como un punto de llegada exclusivamente, ampliando así el espectro de lo que en la literatura más aceptada se considera como una innovación social. Esto se puede explicar mediante la metáfora de un árbol frutal, siendo el árbol la innovación social. Los frutos son el resulta- do final, la formalización sostenible mediante innovación de nuestra economía para el caso estudiado. Por consiguiente si el árbol aún no da los frutos no quiere decir que el árbol no exista, es solo que aún no ha llegado al punto en su crecimiento (evolución) que se lo permita.

Las instituciones gubernamentales en asocio con el sector privado pueden plantearse el propósito de ar-

4 Fuente: Sánchez (2013). 
ticularse para el logro de una innovación social, sin embargo debe ser claro que siendo su objetivo muy superior, la tarea consistirá en construir una red interinstitucional suficientemente sólida hasta el punto que la misma red de instituciones emerja como una nueva organización capaz de proveer todo el patrocinio, recursos y alineamiento estratégico requerido para lograr el retador objetivo trazado; cada uno de los procesos colaborativos que secuencialmente van construyendo la red interinstitucional hace parte de la innovación social hasta finalmente dar su fruto en términos del objetivo superior trazado.

La falta de comprensión de la naturaleza evolutiva de las innovaciones sociales genera frustración en los funcionarios, ciudadanos y organizaciones participantes de los esfuerzos colaborativos al cometer el error de plantearse tempranamente el logro de objetivos muy superiores que no pueden ser alcanzados sin la disponibilidad y fortaleza de las redes interinstitucionales. Un enfoque acertado debería definir un objetivo muy superior (el fruto de la innovación social) y trazar la serie de procesos colaborativos que deberán ir llevando a la red al nivel de disponibilidad y fortaleza requerido para finalmente cumplir dicho objetivo muy superior.

El modelo evolutivo de gobierno colaborativo es una herramienta que sirve para diagnosticar el estado de las redes que se utilizarían como plataforma para el cambio social en innovaciones sociales y a su vez definir estrategias evolutivas para llevar a cabo innovaciones sociales. Esto es, abonar la tierra y seguir unos protocolos para garantizar que el árbol crezca.

Al finalizar este estudio, queda claro para los autores que cualquier innovación social, por su propia naturaleza debe involucrar a los actores del sistema social y por tanto debería ser considerada, por su naturaleza, abierta.

\section{Referencias}

Agranoff, R. (2006). Inside collaborative networks: ten lessons for public managers. En: Public Administration Review, 66: 56-65.

Ansell, C. \& Gash, A. (2007). Collaborative governance in theory and practice. En: Journal of Public Administration Research and Theory, 18: 543-571.

Bloom, P. \& Dees, J. (2008). Cultivate your ecosystem. En: Stanford Social Innovation Review, Winter.
Cabal, M. (2011). Inventario y diagnóstico de la oferta de instrumentos de apoyo a la formalización empresarial y laboral $e$ innovación existentes a nivel internacional y de experiencias sobre la aplicación de dichos instrumentos. Estrategia de apoyo a la formalización empresarial y laboral a partir de actividades de investigación, innovación y desarrollo tecnológico. Colciencias.

Cahill, G. (2010). Primer on social innovation a compendium of definitions developed by organizations around the world. En: The Philanthropist, 23(3).

Cárdenas, M. \& Mejía, C. (2007). Informalidad en Colombia: nueva evidencia. Working Papers Series. Documentos de Trabajo 002858, Fedesarrollo.

Cárdenas, M. \& Rozo, S. (2009). Informalidad empresarial en Colombia: problemas y soluciones. En: Revista Desarrollo y Sociedad, Universidad de Los Andes - Cede.

Castro, J. (2012a). Análisis de las experiencias obtenidas en la aplicación de instrumentos de política pública dirigidos a la formalización y la innovacion. En: Estrategia de apoyo a la formalización empresarial y laboral a partir de actividades deinvestigación, innovación y desarrollo tecnológico, Colciencias.

Castro, J. (2012b). Análisis de la relación entre las estrategias prioritarias de desarrollo implementadas en Santander y las herramientas y proyectos de innovación apoyados en la región. En: Estrategia de apoyo a la formalización empresarial y laboral a partir de actividades deinvestigación, innovación y desarrollo tecnológico, Colciencias.

Castro, J. (2012c). Documento de inventario de los instrumentos nacionales relacionados con la formalización empresarial y laboral e innovación que operacionalizan los lineamientos contenidos en la normatividad y política. En: Estrategia de apoyo a la formalización empresarial y laboral a partir de actividades deinvestigación, innovación y desarrollo tecnológico, Colciencias.

Castro, J. (2012d). Identificación y análisis de las necesidades y oportunidades a partir de la información recogida de las actas de los subcomités y el instrumento elaborado y aplicado. En: Estrategia de apoyo a la formalización empresarial y laboral a partir de actividades deinvestigación, innovación y desarrollo tecnológico, Producto 4.1.3., Colciencias.

Castro, J. (2012e). Inventario y análisis de las estrategias prioritarias de desarrollo de Santander que permita evidenciar los lineamientos contenidos en las estrategias y su consecuente impacto sobre la formalidad y la innovación. En: Estrategia de apoyo a la formalización empresarial y laboral a partir de actividades deinvestigación, innovación y desarrollo tecnológico, Colciencias.

Castro, J. (2012f). Revisión del esquema de funcionamiento y procesos de cada uno de los instrumentos identificados en el inventario de instrumentos. En: Estrategia de apoyo a la formalización empresarial y laboral a partir de actividades deinvestigación, innovación y desarrollo tecnológico, Colciencias.

CEDE (2011). Seminario: la informalidad laboral. Diagnóstico y propuesta de política para Colombia. Universidad de Los Andes, Auditorio ML-A.

Cooper, T., Bryer, T. \& Meek, J. (2006). Citizen-centered collaborative public management. En: Public Administration Review, 66: 76-88.

Chen, M. (2007). Rethinking the informal economy: linkages with the formal economy and the formal regulatory environment. En: Guha-Khasnobis, B., Kanbur, R. \& Ostrom, E. (2007). Linking the formal and informal economy: concepts and policies. Oxford University Press. 
Chenery, H., Ocampo, J. \& Ramírez, M.(1986). El problema laboral colombiano: diagnóstico, perspectivas y políticas. Informe.

Dagnino, G. (2004). Complex systems as key drivers for the emergence of a resource- and capability-based interorganizational network. En: E:CO, 6(1-2): 61-68.

Edelenbos, J. (2005). Institutional implications of interactive governance: insights from dutch practice. En: Governance, 18(1): 111-134.

Emerson, K., Nabatchi, T. \& Balogh, S. (2012). An integrative framework for collaborative governance. En: Journal of Public Administration Research and Theory, 22(1): 1-29.

Fals-Borda, O. (1998). People's participation: challenges ahead. Apex Press.

Gerxhani, K. (2004). The informal sector in developed and less developed countries: a literature survey. En: Public choice, 120(3-4): 267-300.

Goldenberg, M., Kamoji, W., Orton, L. \& Williamson, M. (2009). Social innovation in Canada: an update. CPRN - Research Report: Canadian Policy Research Networks.

Gómez, E. (2013). Capacidades necesarias para el escalamiento de la estrategia de acoplamiento oferta - demanda, como apoyo a la formalización sostenible. Colciencias - Cámara de Comercio de Bucaramanga.

González, J. (2003). Juan Luis Londoño: ideas para la acción. En: Revista de Economía Institucional, 5(8): 223-239.

Guha-Khasnobis, B., Kanbur, R. \& Ostrom, E. (2007). Linking the formal and informal economy: concepts and policies. Oxford University Press.

Hart, K. (1973). Informal income opportunities and urban employment in Ghana. En: The journal of modern African studies, 11(01): 61-89.

Hawamdeh, S. (2007). Creating collaborative advantage through knowledge and innovation. River Edge, NJ, USA: World Scientific.

Hernández, I. (2011). Análisis teórico y conceptual del problema de la informalidad laboral y empresarial. En: Estrategia de apoyo a la formalización empresarial y laboral a partir de actividades deinvestigación, innovación y desarrollo tecnológico, Colciencias.

Hernández, I. (2012a). El análisis institucional de la Aerocivil de Colombia. En: Rodriguez, C. \& Portes, A. (eds). Las instituciones en Colombia: un análisis sociológico. Universidad de Los Andes. Colección estudios Cijus: 161-181.

Hernández, I. (2012b). Propuesta de instrumentos para apoyar la formalización empresarial y laboral a partir de actividades de investigación, innovación y desarrollo tecnológico, como base para la ejecución de la prueba piloto. En: Estrategia de apoyo a la formalización empresarial y laboral a partir de actividades deinvestigación, innovación y desarrollo tecnológico, Colciencias.

Hernández, I. (2013). Formalización sostenible mediante la innovación. En: Silva, J. (comp.) Globalización, crecimiento y desarrollo. Comisión sobre problemas del desarrollo. Bogotá: Academia Colombia de Ciencias Económicas. Colecciones Aportes.

Johnston, E., Hicks, D., Nan, N. \& Auer, J. (2011). Managing the inclusion process in collaborative governance. En: Journal of Public Administration Research and Theory, 21(4): 699-721.

Kania, J. \& Kramer, M. (2011). Collective impact. En: Stanford Social Innovation Review, Winter.

López, H. (1986). La Misión Chenery: una invitación a pensar en el mediano y largo plazo. En: Lecturas de Economía, № 20.

Maloney, W. (2003). Informal self-employment: poverty trap or decent alternative? En: Pathways Out of Poverty: 65-82. Springer Netherlands.
Maloney, W. (2004). Informality revisited. En: World development, 32(7): 1159-1178.

McGuire, M. (2006). Collaborative public management: assessing what we know and how we know it. En: Public Administration Review, 66: 33-43.

Morrison, K. \& Singer, M. (2007). Inequality and deliberative development: revisiting Bolivia's experience with the PRS . En: Development Policy Review, 25(6): 721-740.

Moore, M. \& Westley, F. (2011). Surmountable chasms: networks and social innovation for resilient systems. En: Ecology and Society, 16(1).

Mulgan, G. (2006). The process of social innovation. En: Innovations: Technology, Governance, Globalization, 1(2): 145-162.

Méndez, R., Michelini, J. \& Romeiro, P. (2006). Redes socioinstucionales e innovación para el desarrollo de las ciudades intermedias. En: CIUDAD Y TERRITORIO Estudios Territoriales, XXXVIII(148): 377-395.

Nambisan, S. (2009). Platforms for collaboration. En: Stanford Social Innovation Review, Summer.

Novy, A. \& Leubolt, B. (2005). Participatory budgeting in Porto Alegre: social innovation and the dialectical relationship of state and civil society. En: Urban Studies, 42(11): 2023-2036.

O'Leary, R., Gerard, C. \& Bingham, L. (2006). Introduction to the Symposium on Collaborative Public Management. En: Public Administration Review, 66: 6-9.

Perry, G., Maloney, W., Arias, O., Fajnzylber, P., Mason, A. \& SaavedraChanduvi, J. (2007) Informalidad: escape y exclusion. Banco Mundial. Disponible en: http://siteresources.worldbank.org/INTLACINSPANISH/Resources/SP lacf_Overview.pdfhttp://www. ild.org.pe/idb/colombia.pdf[Consultado: 05 Septiembre 2011

Pol, E. \& Ville, S. (2009). Social innovation: buzz word or enduring term? En: The Journal of Socio-Economics, 38(6): 878-885.

Portes, A. (2009). Las instituciones en el desarrollo latinoamericano: un estudio comparado. Siglo XXI.

Portes, A. (2007). Instituciones y desarrollo: una revisión conceptual. Desarrollo Económico: 475-503.

Revista Semana (1986) Crecer o morir. Entrevista a Hollis Chenery (25 agosto).

Richer, M. (2005). Innovación social y desarrollo local en un municipio andino. En: Revista Venezolana de Economía Social, 5(9): 49-65.

Rodríguez, A. \& Alvarado, H. (2008). Claves de la innovación social en América Latina y el Caribe. Santiago de Chile: Comisión Económica para Amércia Latina y el Caribe (CEPAL). Publicación de las Naciones Unidas

Sánchez, O. (2013). Procesos de gobierno colaborativo en su entorno: una aproximación evolutiva. (Tesis aprobada-Magister en Administración), Universidad Nacional de Colombia, Sede Bogotá.

Santamaría, M. \& Rozo, S. (2009). Análisis cualitativo y cuantitativo de la informalidad empresarial en Colombia. En: Revista Desarrollo y Sociedad, 63(1): 269-296.

Simsek, M. (2014). Sobras y luces de la economía: las ventajas de limitar la informalidad. En: El Espectador. Sección Negocios, 20 de enero. Ministro de Finanzas de Turquía. Traducción de Kena Nequiz. Project Syndicate, disponible en: www.projectsyndicate.org

Sirianni, C. (2008). Investing in democracy: engaging citizens in collaborative governance. Washington, DC, USA: Brookings Institution Press. 
Swyngedouw, E. (2005). Governance innovation and the citizen: the janus face of governance-beyond-the-state. En: Urban Studies, 42(11): 1991-2006.

Taborda, J. (2011a). Inventario de los instrumentos relacionados con la formalización empresarial y laboral e innovación que operacionalizan los lineamientos contenidos en la normativa y política. En: Estrategia de apoyo a la formalización empresarial y laboral a partir de actividades deinvestigación, innovación y desarrollo tecnológico, Colciencias.

Taborda, J. (2011b). Inventario específico de conceptos y lineamientos generales contenidos en los documentos de política vigentes que incentivan la formalización y la innovación empresarial. En: Estrategia de apoyo a la formalización empresarial y laboral a partir de actividades deinvestigación, innovación y desarrollo tecnológico, Colciencias.

Taborda, J. (2011c). Inventario específico de las políticas y normatividad vigente que incentiva la formalización. En: Estrategia de apoyo a la formalización empresarial y laboral a partir de actividades deinvestigación, innovación y desarrollo tecnológico, Colciencias.
Taborda, J. (2011d). Inventario general de las normas y politicas nacionales en materia de innovacion. En: Estrategia de apoyo a la formalización empresarial y laboral a partir de actividades deinvestigación, innovación y desarrollo tecnológico, Colciencias.

Taborda, J. (2011e). Relación entre innovación y formalización en el marco de las políticas de productividad y competitividad: potencial para una política de formalización para la innovación. En: Estrategia de apoyo a la formalización empresarial y laboral a partir de actividades deinvestigación, innovación y desarrollo tecnológico, Colciencias.

Yin, R. (2003). Case study research design and methods (3rd ed.). Thousand Oaks, Calif: Sage.

Zhang, J. \& Cao, Y. (2010). Regional public administration under the framework of collaborative governance. Paper presented at the Management Science and Engineering (ICMSE): 24-26.

Zurbano, M. (2008). Gobernanza e innovación social: el caso de las políticas públicas en materia de ciencia y tecnología en Euskadi. En: Revista de Economía Pública, Social y Cooperativa, CIRIEC-España, 60: 73-93. 